\title{
USO DA TÉCNICA DE SUPERFÍCIE DE RESPOSTA NA AVALIAÇÃO DE PARÂMETROS DO MÉTODO DE ELEMENTOS DISCRETOS NA SIMULAÇÃO DE SECADORES ROTATÓRIOS CONVENCIONAIS COM FERTILIZANTES
}

\author{
B. C. SILVÉRIO ${ }^{*}$, K. G. SANTOS ${ }^{2}$, I. A. RESENDE ${ }^{3}$, M. A. S. BARROZO ${ }^{3}$ \\ ${ }^{1}$ Universidade Federal do Triângulo Mineiro, ICTE/Departamento de Engenharia de Alimentos \\ ${ }^{2}$ Universidade Federal do Triângulo Mineiro, ICTE/Departamento de Engenharia Química \\ ${ }^{3}$ Universidade Federal de Uberlândia, Faculdade de Engenharia Química \\ e-mail: beatrizcristinadejesus@ hotmail.com
}

\begin{abstract}
RESUMO
O Método de Elementos Discretos (DEM) tem se mostrado uma técnica computacional cada vez mais utilizada para simular o comportamento de sistemas em fase discreta em sistemas particulados. Existem vários estudos de DEM para diferentes tipos de secadores e tambores rotativos, porém esses estudos são aplicados para diferentes tipos de materiais. Este trabalho avaliou simulações do escoamento de partículas em secadores rotatórios convencionais utilizando DEM. As simulações foram feitas com diferentes combinações de valores de parâmetros para um modelo linear, e através da comparação dessas simulações com dados experimentais foi possível avaliar o efeito de cada parâmetro e da combinação de parâmetros para o escoamento de fertilizantes granulados. Usando a técnica de superfícies de resposta para o tratamento estatístico dos resultados foi possível verificar a influência de cada parâmetro do modelo linear estudado na massa ou holdup de fertilizantes nos suspensores em diferentes posições angulares. A partir desses valores para melhor compreensão dos resultados de simulação, bem como para quantificação do efeito dos parâmetros e das respectivas interações sobre a previsão da carga nos suspensores, foi feita uma regressão múltipla dos resultados. Os resultados revelam que todos parâmetros estudados foram significativos e houve interação entre os parâmetros relacionados à fricção e ao coeficiente de restituição.
\end{abstract}

\section{INTRODUÇÃO}

Um melhor entendimento dos fenômenos envolvidos na fluidodinâmica de um secador rotativo, necessariamente passa pelo desenvolvimento de uma modelagem com base nas equações de conservação de massa, momento e energia, com suas equações constitutivas pertinentes, bem como pelo acoplamento de pressão e velocidade.

A validação dos modelos empregados pode atenuar problemas de contato sólido-gás, ou mesmo indicar a necessidade de modificação da configuração e de condições operacionais, a fim de propiciar um melhor escoamento das partículas sólidas e um melhor contato gás-partícula.

A resolução numérica dos escoamentos multifásicos pode ser compreendida tanto pela abordagem Euler-Lagrange, quanto pela abordagem Euler-Euler. A abordagem EulerLagrange consiste em modelar a fase fluida como contínua utilizando as equações de Navier-Stokes calculadas no tempo, ausente 
da fase discreta, sendo assim, utiliza-se das informações fluidodinâmicas como dados de entrada para então fazer a descrição do comportamento da fase discreta.

A fase discreta é resolvida injetando-se um grande número de partículas, através do campo de escoamento calculado e são tratadas pela mecânica clássica do corpo sólido, especificamente pela aplicação da $2^{\mathrm{a}}$ Lei do movimento de Newton, sendo considerada a interação de momento, massa e energia da fase discreta com a fase fluida (SANTOS, 2007).

Dentre as dificuldades encontradas na abordagem Lagrangeana, podemos ressaltar a necessidade de utilizar correlações para descrever as interações partícula-parede, partícula-partícula e partícula-gás e a dificuldade em prever as variáveis de campo para a fase particulada, dificultando a visualização científica dos fenômenos que influenciam nas trajetórias das partículas.

Um método aplicado para a abordagem Lagrangeana consiste na utilização de modelos DEM (Discret Element Method) ou Método dos Elementos Discretos, que permite simular partículas em movimento como pontos de massa em sistemas concentrados.

Di Renzo (2004) comparou três modelos para forças de contato, e numa comparação macroscópica o modelo linear "mola-amortecedor" (spring-dashpot) estudado mostrou bons resultados para a predição das forças de contato.

A literatura mostra vários trabalhos de estudos e verificações de modelos que se utilizam de vários parâmetros, dependendo do processo e material analisados (ANAND et al., 2009; KETTERHAGENG et al., 2008; LIU, YANG, YU, 2013; ZHONG et al., 2006 e GENG et al., 2009). Alguns dos parâmetros utilizados, por exemplo, no modelo linear "mola-amortecedor", citado anteriormente, são os coeficientes de restituição $(\eta)$, de fricção $(\mu)$ e o coeficiente de elasticidade ou mola $(k)$.
Nota-se que os valores encontrados para os parâmetros dos modelos nos trabalhos de simulação DEM, variam significativamente, pois cada estudo foi concebido para equipamentos distintos e representa o escoamento de materiais diferentes os quais podem conduzir diferentes respostas de colisões partícula-partícula e partícula-parede.

Desta forma, o objetivo deste trabalho foi realizar simulações utilizando o método DEM e avaliar diferentes valores dos parâmetros: coeficientes de restituição $(\eta)$, de fricção $(\mu)$ e o coeficiente de elasticidade ou mola $(k)$ do modelo linear "molaamortecedor" para encontrar um melhor conjunto de parâmetros que melhor caracterize o sistema em estudo.

\section{METODOLOGIA}

\subsection{Procedimento Experimental}

Foi utilizado um secador rotatório convencional com $0,3 \mathrm{~m}$ de diâmetro, $1,5 \mathrm{~m}$ de comprimento, contendo 6 suspensores de 3 segmentos de $0,7 \times 0,7 \times 2,0 \mathrm{~cm}$.

Foi introduzida uma massa de $4,75 \mathrm{~kg}$ de fertilizante superfosfato simples granulado (SSPG), por uma calha semicircular com o equipamento posicionado com um dos suspensores na posição angular $0^{\circ}$. A Figura 1 mostra o equipamento no tempo zero.

A rotação do equipamento era ligada em 3,6 rpm e o equipamento foi fotografado com a finalidade de se obter o ângulo de repouso e a área de ocupação do fertilizante nos suspensores durante a segunda volta do experimento, ou seja durante os 20 primeiros segundos.

As imagens foram analisadas e os ângulos de repouso dos suspensores foram obtidos a partir do software GLOBAL Lab® IMAGE/2. As áreas de preenchimento dos suspensores também foram obtidas para o cálculo do volume de partículas em cada suspensor nas posições angulares analisadas a partir do software GLOBAL Lab® IMAGE/2. 
Figura 1 - Fertilizante introduzido no início de cada experimento.

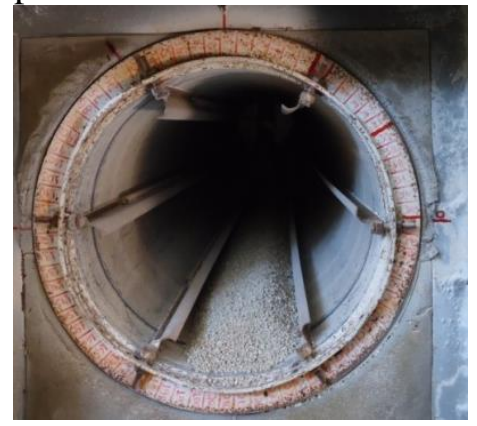

A área então foi multiplicada pelo comprimento do suspensor (1,5 m) e então se descontando a porosidade $(43 \%)$ obteve-se o volume e massa de fertilizantes em cada suspensor pela técnica de análise de imagem.

Após a obtenção das imagens o equipamento foi esvaziado e novamente preenchido com a mesma massa de $4,75 \mathrm{~kg}$ de fertilizantes para obtenção experimental das massas ou carga de fertilizantes nos suspensores.

A massa de partículas nos suspensores em função da sua posição angular no tambor foi efetuada por meio de paradas repentinas do secador em diversas posições angulares e posterior coleta do material durante a segunda volta do experimento. Isto permitiu que os valores de carga de sólidos dos suspensores (h) pudessem ser comparados com os obtidos no mesmo tempo simulado. Foram retiradas as cargas de sólidos nos suspensores durante a segunda volta do experimento para as posições angulares de $0^{\circ}, 30^{\circ}, 60^{\circ}, 90^{\circ}$ e $120^{\circ}$..

\subsection{Metodologia de Simulação}

O software FLUENT® 14.0 foi utilizado nas simulações para a obtenção do ângulo dinâmico de repouso e da carga de partículas nos suspensores na avaliação dos parâmetros do Método dos Elementos Discretos (DEM) secadores rotativos.

A malha computacional foi obtida a partir do software GAMBIT® 2.3.16 e foi construída de forma a se garantir que o tamanho das células fosse no mínimo três vezes maior do que o diâmetro da esfera de igual volume da partícula $(d p)$.

Uma vez que se opta pela utilização do modelo Euleriano, o software FLUENT ANSYS $® 14.0$, além de resolver as equações de transporte para a fase contínua, permite simular uma fase discreta segundo uma abordagem Lagrangeana que utiliza o DEM (Discret Element Method). Esta segunda fase consiste em partículas esféricas. O acoplamento entre as fases e o seu impacto sobre ambas as trajetórias da fase discreta e o fluxo de fase contínua também é incluído nos cálculos.

O modelo utilizado resolve para a fase fluida a equação da Continuidade e a equação do Movimento.

As simulações DEM foram feitas utilizando-se malhas construídas em três dimensões. O conjunto de equações de conservação e constitutivas foi resolvido utilizando-se o método dos volumes finitos. O acoplamento entre velocidade e pressão foi definido pelo algoritmo SIMPLE. Os resíduos utilizados foram menores que $10^{-3}$. Na parede foi considerada uma condição de contorno de não deslizamento. Para a discretização espacial foi utilizado o modelo QUICK e para a discretização temporal o modelo implícito de primeira ordem.

Nas simulações do secador rotatório com suspensores foi necessário utilizar uma condição de malha móvel, em que toda a malha se movimenta à uma mesma velocidade rotacional. $\mathrm{O}$ modelo mais geral para o fluxo em zonas móveis com deformação celular no FLUENT ANSYS ${ }^{\circledR}$ é o modelo de malha móvel.

A fim de avaliar o efeito dos parâmetros do modelo DEM sobre o ângulo dinâmico de repouso nos suspensores, bem como na carga de fertilizantes nos suspensores, foram realizadas simulações previstas por meio de um planejamento composto central ortogonal 
(PCC) com 7 réplicas no ponto central, conforme mostra a Tabela 1 (simulação 15 são os valores do ponto central), (BOX, M. J., HUNTER, W. G. E HUNTER, J. S., 1978).

Tabela 1 - Planejamento composto central para avaliação dos parâmetros.

\begin{tabular}{cccc}
\hline Simulação & $k[\mathrm{~N} / \mathrm{m}]$ & $\mu_{f}$ & $\eta$ \\
\hline $\mathbf{1}$ & 400 & 0,2000 & 0,2000 \\
$\mathbf{2}$ & 400 & 0,2000 & 0,8000 \\
$\mathbf{3}$ & 400 & 0,8000 & 0,2000 \\
$\mathbf{4}$ & 400 & 0,8000 & 0,8000 \\
$\mathbf{5}$ & 1600 & 0,2000 & 0,2000 \\
$\mathbf{6}$ & 1600 & 0,2000 & 0,8000 \\
$\mathbf{7}$ & 1600 & 0,8000 & 0,2000 \\
$\mathbf{8}$ & 1600 & 0,8000 & 0,8000 \\
$\mathbf{9}$ & $\mathbf{5 4 , 9 8}$ & 0,5000 & 0,5000 \\
$\mathbf{1 0}$ & $\mathbf{1 9 4 5}$ & 0,5000 & 0,5000 \\
$\mathbf{1 1}$ & 1000 & $\mathbf{0 , 0 2 7 5}$ & 0,5000 \\
$\mathbf{1 2}$ & 1000 & $\mathbf{0 , 9 7 2 5}$ & 0,5000 \\
$\mathbf{1 3}$ & 1000 & 0,5000 & $\mathbf{0 , 0 2 7 5}$ \\
$\mathbf{1 4}$ & 1000 & 0,5000 & $\mathbf{0 , 9 7 2 5}$ \\
$\mathbf{1 5}$ & 1000 & 0,5000 & 0,5000
\end{tabular}

Os valores dos parâmetros escolhidos tiveram como base a ampla faixa encontrada na revisão da literatura. Sendo assim, nem todas as combinações entre os parâmetros necessariamente conduzem a resultados fisicamente coerentes, mas pode contribuir para verificar o efeito dos parâmetros e suas interações.

A Tabela 2 apresenta as condições utilizadas nas simulações do ângulo dinâmico de repouso e da carga de partículas de fertilizantes nos suspensores do secador rotatório convencional.

Para diminuir o tempo de processamento a simulação do secador rotatório, optou-se por utilizar uma malha que representasse uma fatia de um décimo do comprimento do tambor do secador rotatório, ou seja, uma malha com as mesmas dimensões de diâmetro, porém com comprimento igual a $0,15 \mathrm{~m}$.

Tabela 2 - Condições adotadas nas simulações do secador rotatório convencional.

$\begin{array}{cc}\text { time step fase contínua }(\mathrm{s}) & \mathbf{0 , 0 0 1} \\ \text { time step fase discreta }(\mathrm{s}) & \mathbf{0 , 0 0 0 1} \\ \text { Velocidade rotacional }(\mathrm{rpm}) & \mathbf{3 , 6} \\ \mathrm{N}^{\circ} \text { de partículas } & \mathbf{2 0 5 8 3} \\ \mathrm{N}^{\circ} \text { de células da malha } & \mathbf{9 3 3 8} \\ \text { Tempo simulado }(\mathrm{s}) & \mathbf{3 3} \\ \mathrm{N}^{\circ} \text { de suspensores } & \mathbf{6} \\ \text { Diâmetro do tambor }(\mathrm{m}) & \mathbf{0 , 3 0} \\ \text { Diâmetro da partícula }(\mathrm{dp})(\mathrm{m}) & \mathbf{0 , 0 2 8 8} \\ \text { Comprimento do tambor }(\mathrm{m}) & \mathbf{0 , 1 5}\end{array}$

Nestas simulações foram avaliadas as massas de partículas nos suspensores contidos nas posições angulares de $0^{\circ}, 30^{\circ}, 60^{\circ}, 90^{\circ} \mathrm{e}$ $120^{\circ}$, as mesmas posições avaliadas experimentalmente. Para tal, foram obtidas as massas das partículas em cada posição de suspensor desejada, para as simulações previstas pelo PCC (Tabela 1).

Foram também avaliados os ângulos de repouso dentro de cada suspensor nas posições analisadas. Os valores simulados de massa nos suspensores e os ângulos de repouso foram então comparados com os valores obtidos experimentalmente nas mesmas condições.

Além de comparar os resultados de massa e ângulo nos suspensores obtidos nas simulações aos dados experimentais, a escolha dos parâmetros de simulação que melhor caracterizam o escoamento do fertilizante em tambores rotativos baseou-se também na comparação visual qualitativa entre as imagens obtidas durante o experimento e os perfis simulados de posição das partículas.

\section{RESULTADOS E DISCUSSÕES}

A Tabela 3 apresenta os resultados das massas ou carga de partículas de fertilizante retidas nos suspensores para cada simulação do PCC da Tabela 1. Observa-se que dentre as 
simulações do PCC os menores desvios tanto do ângulo dinâmico de repouso, quanto para a massa ou carga retida nos suspensores $\left(h^{*}\right)$, foram obtidos com os valores dos parâmetros definidos nas simulações 1,2 e 8 ; sendo que dentre estas, a simulação 1 destaca-se por apresentar menores desvios de $h^{*}$.

Tabela 3 - Resultados dos desvios das massas das simulações em relação as massas experimentais em cada posição angular $(\theta)$ dos suspensores.

\begin{tabular}{|c|c|c|c|c|c|c|c|c|}
\hline \multirow[b]{2}{*}{ posição $\theta\left({ }^{\circ}\right)$} & \multicolumn{4}{|c|}{ Massa nos suspensores $\left(h^{*}\right)(\mathrm{g})$} & \multicolumn{4}{|c|}{ Erro em relação ao experimento $(\%)$} \\
\hline & $\mathbf{0}$ & 30 & 60 & 90 & $\mathbf{0}$ & 30 & 60 & 90 \\
\hline experimento & 1124,4 & 590,7 & 407,1 & 149,6 & - & - & - & - \\
\hline simulação 01 & 1115,75 & 691,61 & 396,92 & 101,99 & 0,8 & 4,2 & 2,5 & 31,8 \\
\hline simulação 02 & 984,68 & 641,99 & 363,69 & 78,46 & 12,4 & 11,1 & 10,7 & 47,5 \\
\hline simulação 03 & 1676,75 & 863,53 & 802,60 & 328,84 & 49,1 & 19,6 & 97,1 & 120,0 \\
\hline simulação 04 & 1326,90 & 833,30 & 563,76 & 267,23 & 18,0 & 15,4 & 38,5 & 78,7 \\
\hline simulação 05 & 765,91 & 561,68 & 288,46 & 84,00 & 31,9 & 22,2 & 29,2 & 43,8 \\
\hline simulação 06 & 756,91 & 545,30 & 218,30 & 47,31 & 32,7 & 24,5 & 46,4 & 68,4 \\
\hline simulação 07 & 1440,44 & 887,06 & 623,76 & 330,46 & 28,1 & 22,9 & 53,2 & 121,0 \\
\hline simulação 08 & 1113,91 & 765,91 & 524,30 & 240,00 & 0,9 & 6,1 & 28,8 & 60,5 \\
\hline simulação 09 & 1354,83 & 835,37 & 562,61 & 273,46 & 20,5 & 15,7 & 38,2 & 82,9 \\
\hline simulação 10 & 206,77 & 226,38 & 76,85 & 69,46 & 81,6 & 68,6 & 81,1 & 53,5 \\
\hline simulação 11 & 871,60 & 503,53 & 215,07 & 25,62 & 22,5 & 30,3 & 47,2 & 82,9 \\
\hline simulação 12 & 1366,60 & 841,37 & 582,22 & 284,53 & 21,5 & 16,5 & 43,0 & 90,3 \\
\hline simulação 13 & 156,23 & 170,54 & 30,92 & 58,38 & 86,1 & 76,4 & 92,4 & 60,9 \\
\hline simulação 14 & 1259,06 & 756,91 & 494,07 & 206,77 & 12,0 & 4,8 & 21,4 & 38,3 \\
\hline simulação 15 & 1330,83 & 816,45 & 544,61 & 246,92 & 18,4 & 13,1 & 33,8 & 65,2 \\
\hline simulação 16 & 1345,83 & 815,30 & 549,68 & 242,77 & 19,7 & 12,9 & 35,0 & 62,4 \\
\hline simulação 17 & 1344,90 & 846,68 & 560,30 & 265,38 & 19,6 & 17,3 & 37,6 & 77,5 \\
\hline simulação 18 & 1293,21 & 846,91 & 523,84 & 256,84 & 15,0 & 17,3 & 28,7 & 71,8 \\
\hline simulação 19 & 1363,13 & 808,83 & 552,92 & 239,07 & 21,2 & 12,0 & 35,8 & 59,9 \\
\hline simulação 20 & 1316,75 & 817,83 & 550,38 & 243,00 & 17,1 & 13,3 & 35,2 & 62,5 \\
\hline simulação 21 & 1355,52 & 818,07 & 548,53 & 228,69 & 20,6 & 13,3 & 34,7 & 53,0 \\
\hline
\end{tabular}

$\mathrm{O}$ valor encontrado como melhor para o coeficiente de fricção $\left(\mu_{f}=0,2\right)$ foi próximo da grande maioria dos trabalhos da literatura (ZHONG et al., 2006, GENG et al., 2009).
A Figura 2 mostra os resultados da simulação 01 e 02 , que apresentaram os menores desvios, juntamente com os resultados da técnica de análise de imagem 
experimental, bem como os dados suspensores. Observa-se que os resultados dessas simulações tiveram uma boa concordância com os dados experimentais de

Figura 2 - Massa de fertilizantes $\left(h^{*}\right)$ nas posições angulares dos suspensores.

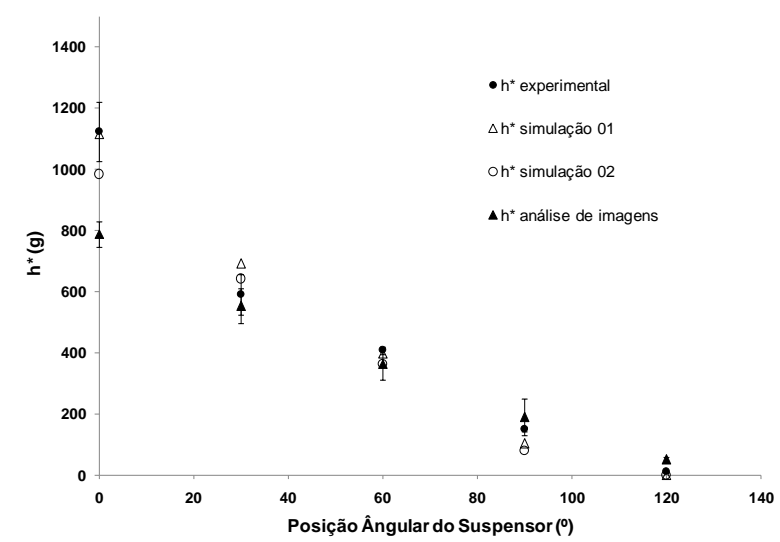

Sendo assim, para melhor compreensão dos resultados de simulação, bem como para quantificação do efeito dos parâmetros e das respectivas interações sobre a previsão da carga nos suspensores, foi feita uma regressão múltipla dos resultados das simulações nos secadores convencionais com suspensores. Nesta regressão foi também introduzida como variável $\left(\mathrm{x}_{4}\right)$ a posição angular, além dos parâmetros analisados.

O modelo apresentou resíduos aleatoriamente distribuídos, com média zero e variância constante. $\mathrm{O}$ valor de $\mathrm{R}^{2}$ foi igual a 0,982 , indicando que $98,2 \%$ da variabilidade da carga nos suspensores foi explicada pelas variáveis analisadas.

A Tabela 4 mostra as variáveis que foram significativas para um nível de significância de 5\% (probabilidade máxima de erro do teste estatístico).

Os resultados da Tabela 4 mostram que todos parâmetros estudados foram significativos e houve interação entre os parâmetros relacionados à fricção e ao coeficiente de restituição. Observa-se também que o parâmetro do modelo DEM que exerceu experimentais de massa de sólidos retida nos $h^{*}$ (medido pela massa), sendo os desvios equivalentes aos da técnica de análise de imagem experimental.

a maior influência foi o coeficiente de fricção $\left(\mu_{f}\right)$ e o de menor influência foi o coeficiente de restituição $(\eta)$.

Tabela 4 - Resultados da regressão múltipla para os parâmetros avaliados.

\begin{tabular}{|c|c|c|c|}
\hline $\begin{array}{c}\text { Variáveis } \\
\text { codificadas }\end{array}$ & Coeficientes & $\begin{array}{c}\text { desvios } \\
(\%)\end{array}$ & $\mathbf{p}$ \\
\hline Coef. Linear B & 51.6613 & 1.285521 & 0.000000 \\
\hline$k\left(x_{1}\right)$ & -5.2147 & 0.948015 & 0.000000 \\
\hline$\mu_{f}\left(x_{2}\right)$ & 12.1565 & 0.793429 & 0.000000 \\
\hline$\eta\left(x_{3}\right)$ & -4.4880 & 0.895545 & 0.000003 \\
\hline$\theta\left(x_{4}\right)$ & -28.9812 & 0.466300 & 0.000000 \\
\hline$x_{1}^{2}$ & -2.9521 & 1.056471 & 0.006441 \\
\hline$x_{2}^{2}$ & -5.1313 & 0.858027 & 0.000000 \\
\hline$x_{4}^{2}$ & 4.2150 & 0.391633 & 0.000000 \\
\hline$x_{1} \cdot x_{4}$ & 2.1780 & 0.627836 & 0.000826 \\
\hline$x_{2} \cdot x_{3}$ & -2.9319 & 1.009928 & 0.004719 \\
\hline$x_{2} \cdot x_{4}$ & -3.3469 & 0.561039 & 0.000000 \\
\hline
\end{tabular}

Equação 1.

O modelo obtido é mostrado na

$$
\begin{aligned}
& h^{*}=51,66-5,21 x_{1}+12,15 x_{2}-4,48 x_{3}-28,98 x_{4}-2,95 x_{1}^{2}-5,13 x_{2}^{2}+ \\
& 4,21 x_{4}^{2}+2,17 x_{1} x_{4}-2,93 x_{2} x_{3}-3,35 x_{2} x_{4}
\end{aligned}
$$

As equações das variáveis codificadas foram as Equações 2, 3, 4 e 5.

$$
\begin{aligned}
& x_{1}=(k-100) / 600 \\
& x_{2}=\left(\mu_{f}-0,5\right) / 0,3 \\
& x_{3}=(\eta-0,5) / 0,3
\end{aligned}
$$




\section{O. ENEMP 2015 São Carlos - SP}

$$
x_{1}=(\theta-60) / 30
$$

A Figura 3 mostra a superfície de respostas feitas para a posição angular $\theta=$ $0^{o}$ para $k=400$ (equivalente ao nível codificado -1), o melhor resultado.

Figura 3 - Superfície de resposta na posição $\theta=0^{\circ}$ e para $k=400$.

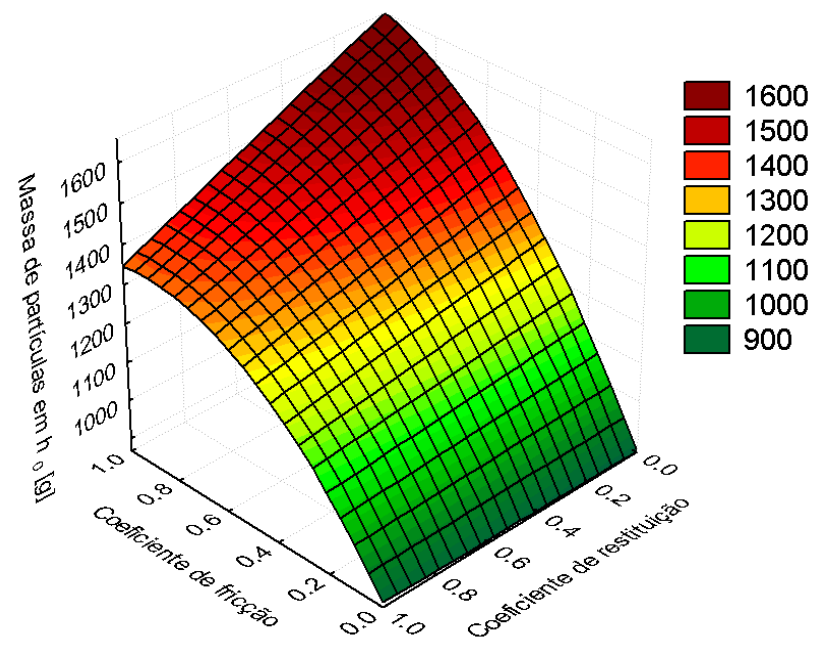

A Figura 4 mostra a superfície de respostas feitas para a posição angular $\theta=$ $0^{o}$ para $k=1600$ (equivalente ao nível codificado 1).

Figura 4 - Superfície de resposta na posição $\theta=0^{\circ}$ e para $k=1600$.

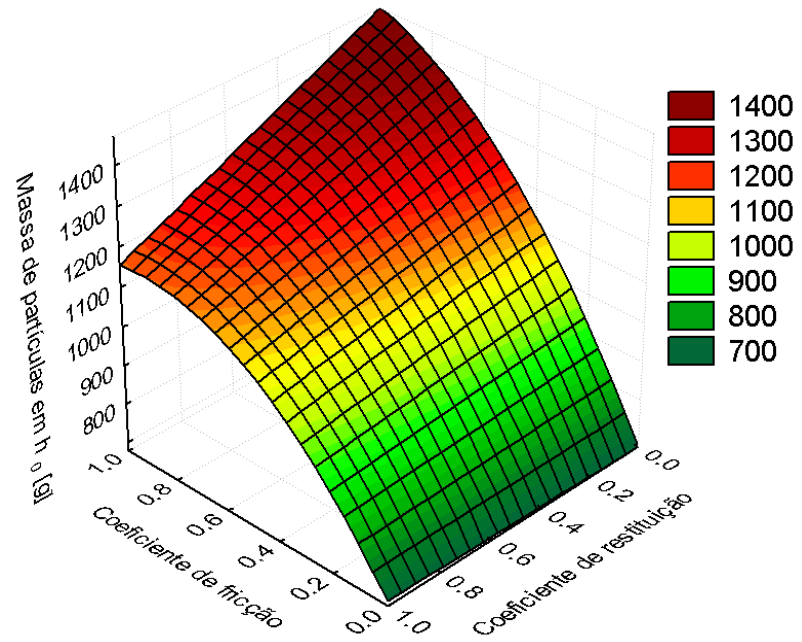

A Figura 5 mostra a superfície de respostas feitas para a posição angular $\theta=0^{\circ}$ para o valor de $\mu_{f}=0,2$ (equivalente ao nível codificado -1 , o melhor resultado).

Figura 5 - Superfície de resposta na posição $\theta=0^{\circ}$ e para $\mu_{f}=0,2$.

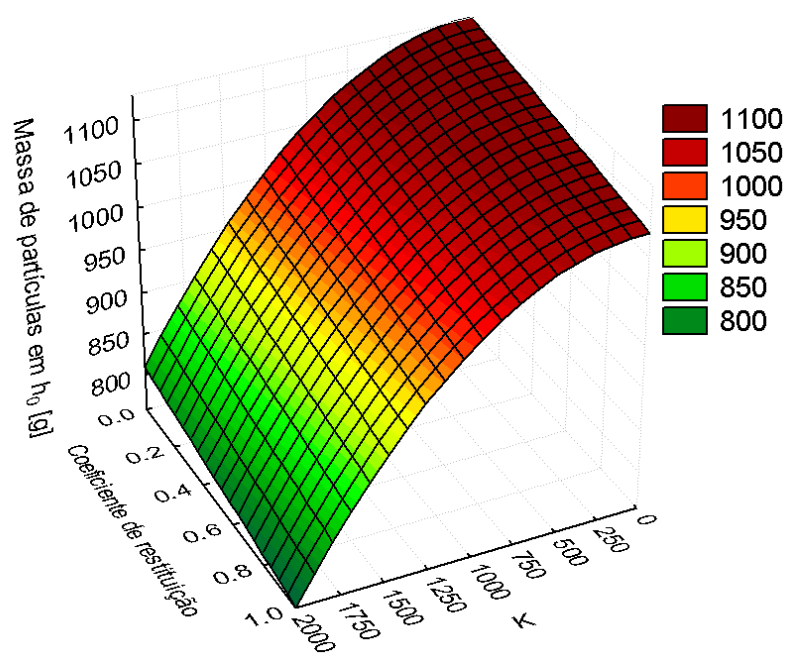

A Figura 6 mostra a superfície de respostas feitas para a posição angular $\theta=0^{\circ}$ para o valor de $\mu_{f}=0,8$ (equivalente ao nível codificado 1).

Figura 5 - Superfície de resposta na posição $\theta=0^{\circ}$ e para $\mu_{f}=0,8$. 


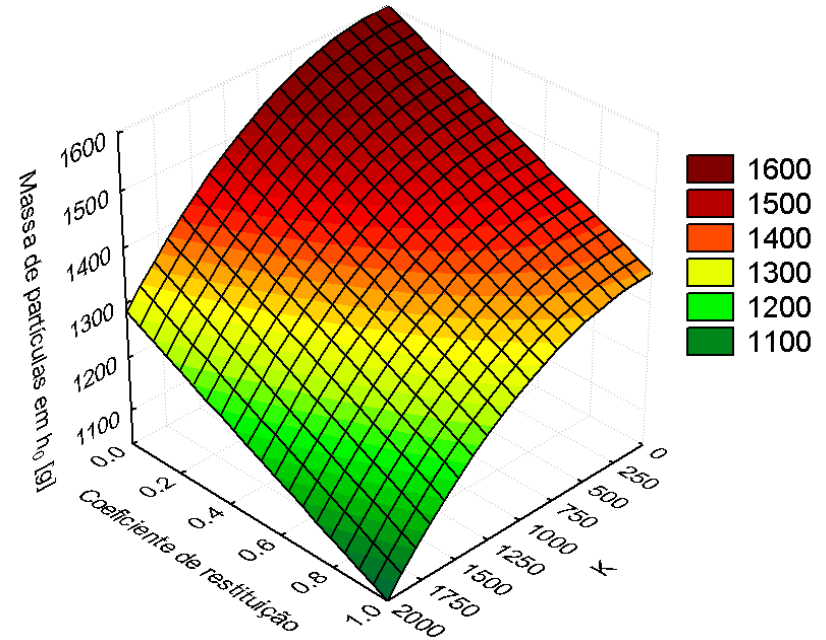

Observa-se pelas Figuras 3 e 4 que para menores valores do coeficiente de fricção $\left(\mu_{f}\right)$ houve pouca influência do aumento do coeficiente de restituição, já em valores maiores de $\mu_{f}$ a variação da resposta com o coeficiente de restituição foi notável, mostrando uma forte interação entre os parâmetros relacionados ao coeficiente de fricção e de restituição. Os maiores valores de carga nos suspensores foram obtidos para baixos níveis do coeficiente de restituição e elevados do coeficiente de fricção, para os dois valores de $k$ apresentados.

Os resultados das Figuras 5 e 6 evidenciam a ausência de interação entre os parâmetros relacionados ao coeficiente de elasticidade $k$ e o coeficiente de restituição $(\eta)$. Além disso, verifica-se a mais expressiva influência do parâmetro relacionado à elasticidade na resposta quando comparada à do parâmetro relacionado ao coeficiente de restituição.

\section{CONCLUSÕES}

Os resultados apresentados mostraram que a técnica de tratamento estatístico de dados usando superfície de resposta se mostrou uma importante ferramenta para a compreensão dos resultados de simulação
DEM para diferentes parâmetros do modelo linear.

Foi possível verificar através das superfícies de resposta a forte interação entre os parâmetros relacionados ao coeficiente de fricção e de restituição.

Além disso verificou-se a mais expressiva influência do parâmetro relacionado à elasticidade na resposta (carga nos suspensores) quando comparada à do parâmetro relacionado ao coeficiente de restituição.

\section{NOMENCLATURA}

\section{$\underline{\text { Símbolos }}$}

$h^{*} \quad$ carga ou holdup nos suspensores.

$k \quad$ coeficiente de elasticidade

\section{Letras Gregas}

$\mu_{f} \quad$ coeficiente relacionado a fricção

$\eta \quad$ coeficiente de restituição

\section{REFERÊNCIAS}

ANAND, A.; CURTIS, J. S.; WASSGREN, C. R.; HANCOCK, B.C.; KETTERHAGEN, W.R. Predicting discharge dynamics of wet cohesive particles from a rectangular hopper using the discrete element method (DEM). Chemichal Engineering Science, v. 64, p. 5268-5275, 2009.

BOX, M. J., HUNTER, W. G. E HUNTER, J. S. Statistics for Experimenters. An Introduction to Design, Data Analysis, and Model Building. John Wiley and Sons: New York, 1978.

DI RENZO, A.; DI MAIO, F. P. Comparison of contact-force models for the simulation of collisions in DEM-based granular flow codes. 
Chemical Engineering Science, v. 59, p. 525 - 541, 2004.

GENG F.; YUAN, Z.; YAN, Y.; LUO, D.; WABG, H.; LI, B., CHYANG, C. S. Numerical simulation on mixing kinetics of slender particles in a rotary dryer. Powder Technology, v. 193, p. 50-58, 2009.

KETTERHAGENA, W. R.; CURTIS, J. S.; WASSGREN, C. R.; HANCOCK, B. C. Modeling granular segregation in flow from quasi-three-dimensional wedge shaped hoppers. Powder Technology. v. 179, p. 126143, 2008.

LIU, P. Y.; YANG, N.; YU, A. B. DEM study of the transverse mixing of particles in rotating drums. Chemical Engineering

Science, v. 86, p. 99-107, 2013.

LI, Y., XU, Y.; JIANG, S. DEM simulations and experiments of pebble flow with monosized spheres. Powder Technolology, v. 193 , p. 312-318, 2009.

SANTOS, D. A. Contribuições ao estudo da Fluidodinâmica em leito de jorro - Estudos experimentais e de simulação via CFD. 2007. 107p. Dissertação (Mestrado em Engenharia Química) -Universidade Federal de Uberlândia, Uberlândia - MG, 2007.

ZHONG, W.; XIONG, Y.; YUAN, Z.; ZHANG, M.. DEM simulation of gas-solid flow behaviors in spouted-fluid bed. Chemichal Engineering Science, v. 61, p. 1571-1584, 2006. 